Textures and Microstructures, 1988, Vol. 10, pp. 49-58

Reprints available directly from the publisher

Photocopying permitted by license only

(C) 1988 Gordon and Breach Science Publishers Inc.

Printed in the United Kingdom

\title{
Orientation Maps for Cubic Crystal Neighbours
}

\author{
W. PRANTL and E. WERNER \\ Erich-Schmid-Institut für Festkörperphysik der Österreichischen Akademie \\ der Wissenschaften und Institut für Metallphysik der Montanuniversität \\ Leoben, 8700, Austria
}

(Received 20 January, 1988)

In texture analysis measured orientations are frequently represented in the Euler space. To detect special orientations $(h k l)[u v w]$ use can be made of plane sections (Euler angle $\varphi_{2}=$ const) through the Euler space. Orientation relationships appear as points in these "maps." For texture analysis the points are plotted in maps for cubic crystals in an orthorhombic specimen. In this paper similar maps are proposed to detect special orientation relationships between neighbouring grains in a polycrystal. These maps, based on the symmetry of the Euler space resulting from the cubic symmetry of both crystal neighbours, are discussed and their handling is demonstrated using measured orientation relationships in single and dual-phase brasses.

KEY WORDS: Orientation relationships, orientation maps, cubic symmetry.

\section{INTRODUCTION}

In texture analysis special orientations are frequently represented by two sets of indices $(h k l)$ [uvw]. In case of rolling textures, for example, $(h k l)$ means the crystal plane which is parallel to the specimen surface, while [uvw] specifies the crystal direction which is parallel to the rolling direction. Following an idea of Schläfer and Bunge (1974) these special orientations can be represented graphically in the Euler space. In the literature such maps can be found for the case of cubic crystals in a specimen of orthorhombic symmetry as plane sections through the Euler space. 
Investigating the orientation relationships between neighbouring crystals of cubic symmetry in a specimen one can represent the measured orientation relationships as points in the Euler space. This suggests the use of maps containing special orientations for a qualitative description which allows to indicate parallelism of planes and directions. Since the symmetry of the Euler space is determined by the symmetries of the considered neighbouring crystals, the maps mentioned above, which are conceived for cubic/orthorhombic partners, are not suitable for this purpose.

It is the aim of this paper to introduce maps showing special orientation relationships in the Euler space for neighbouring crystals both belonging to the cubic system and to compare them with maps of cubic/orthorhombic symmetry.

\section{DESCRIPTION OF THE ORIENTATION MAPS}

In a system of cubic symmetry there are 24 possibilities to define a right-handed coordinate system, whereas there are only 4 in an orthorhombic one. Combining two systems of these symmetries gives $24 \times 4=96$ different possibilities to describe the very same physical orientation relationship, that means 96 points in the Euler space. Considering symmetry relations Bunge (1982) has shown that the Euler space can be subdivided into 32 equivalent partial spaces of $90^{\circ} \times 90^{\circ} \times 90^{\circ}$ dimension. So we can restrict our considerations to the partial space $P_{1}$ next to the origin. Each of the partial spaces contains $96 / 32=3$ equivalent points. Considering the case of two partners of cubic symmetry we get $24 \times 24=576$ equivalent points in the Euler space. That means $576 / 32=18$ equivalent points in $P_{1}$. Additionally a mirror plane occurs in each partial space (Werner and Prantl 1988). So, in principle, one could restrict the attention to the half of $P_{1}$ only.

Figures $1 \mathrm{a}$ and $\mathrm{b}$ show 9 sections $\left(\varphi_{2}=\right.$ const) through $P_{1}$ for cubic crystal neighbours. These maps are plotted for planes with indices not greater than 3. Comparison with the maps for cubic/orthorhombic symmetry shows that all points of those maps are also contained in the cubic/cubic maps. While in the cubic/orthorhombic case the direction indices are $\leq 3$, we can find indices $>3$ in the cubic/cubic case. This results from the symmetry of the second 

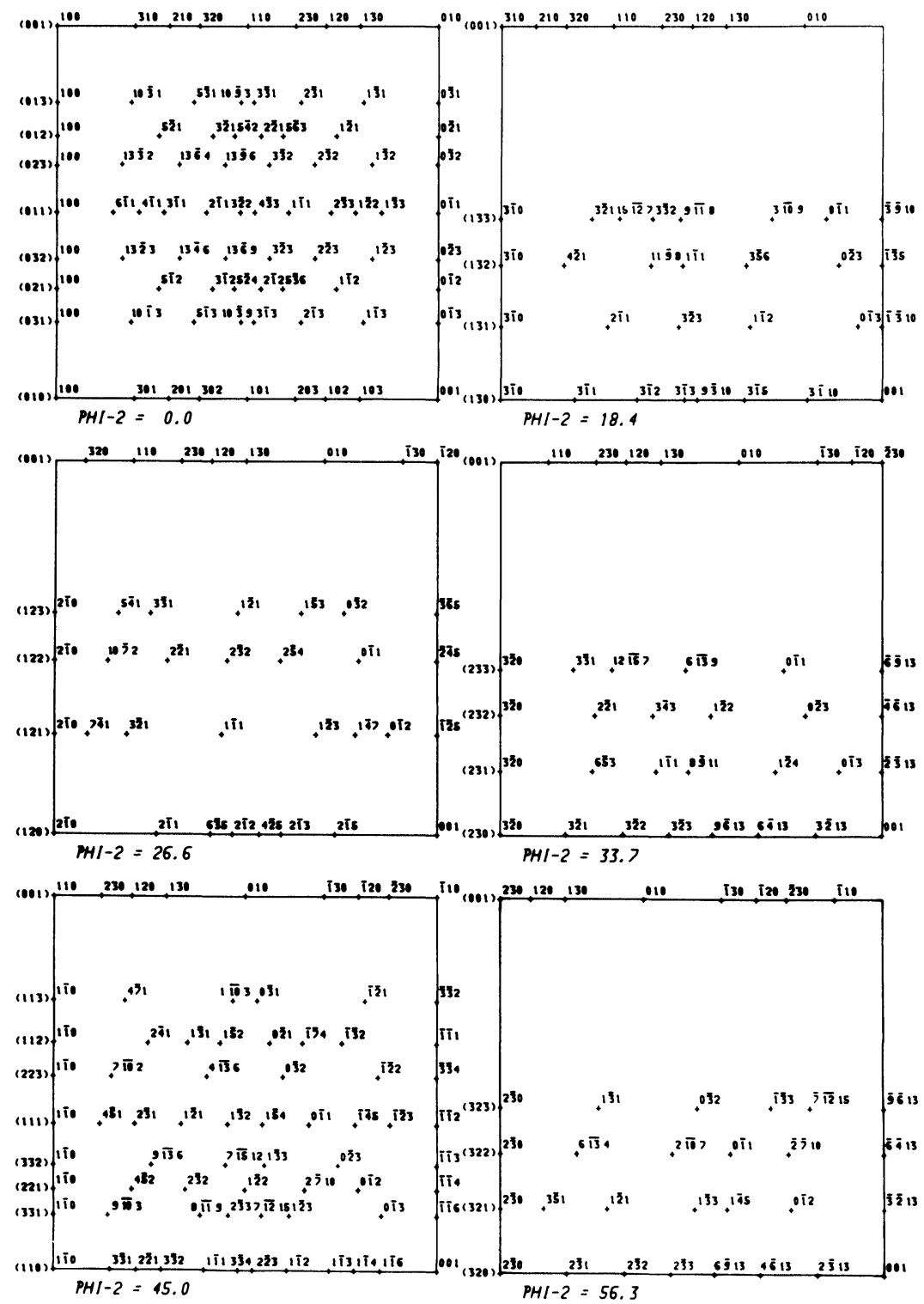

Figure 1a Orientation maps for cubic crystal neighbours $\left(\varphi_{2} \leq 56.3^{\circ}\right.$, plane indices $\leq 3$ ). 

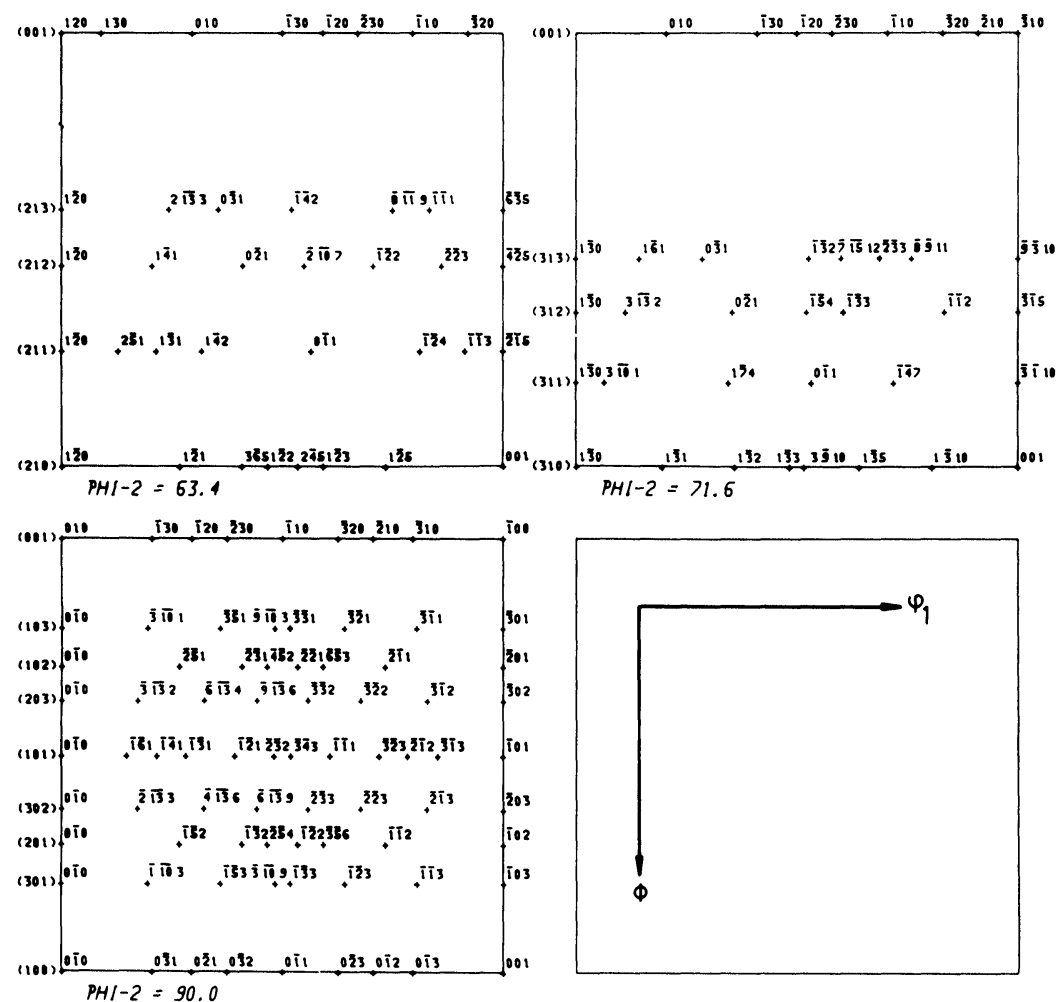

Figure 1b Orientation maps for cubic crystal neighbours $\left(\varphi_{2}>56.3^{\circ}\right.$, plane indices $\leq 3$ ).

partner, which now exhibits a 4-fold rotation axis. This means that for any direction $[u v w]$, lying in the plane $(h k l)$, the direction $[u \perp v \perp w \perp]=[h k l] \times[u v w]$, perpendicular to $[u v w]$, is also parallel to a coordinate axis of the second crystal. Therefore also the index combination $(h k l)[u \perp v \perp w \perp]$ describes the very same physical orientation relationship and gives an equivalent point in the Euler space. The indices of $[u \perp v \perp w \perp]$ can thereby attain values $>3$.

Of course the combination ( $h k l)[u \perp v \perp w \perp]$ would lead to a point in the Euler space also in the case of cubic/orthorhombic symmetry. But that point would by no means be equivalent to the 
starting point $(h k l)[u v w]$ because the coordinate axes of the reference system ( = specimen sheet) in that case are only of 2-fold symmetry. Therefore $(h k l)[u \perp v \perp w \perp]$ would describe a quite different physical orientation relationship.

For illustration let us consider the following example: Let a point in the Euler space be given by (123)[032]. That means that the plane (123) of the first crystal is parallel to a cube plane and the direction [032] is parallel to a cube edge of the second crystal. A second direction in the plane (123) being also perpendicular to a cube edge of the second crystal is easily obtained as [ [ $\left.\begin{array}{lll}13 & \overline{2} & \overline{3}\end{array}\right]$, namely by evaluating the vector product between the plane normal [123] and the first direction [032]. In the case of cubic/cubic symmetry the combination (123) [ [ $\left.\begin{array}{lll}13 & \overline{2} & \overline{3}\end{array}\right]$ therefore characterizes a point in the Euler space being equivalent to the initially given point and describing the same physical orientation relationship.

From Figure 2 we recognize furthermore that also the planes $(0 \overline{3} 2)$ and $\left(\begin{array}{lll}13 & \overline{2} & \overline{3}\end{array}\right)$ are parallel to a cube plane of the second crystal. Since each of these planes again contains two mutually perpendicular directions being parallel to cube edges of the second crystal, we obtain 4 additional equivalent points in the Euler space in this way. Therefore, starting from the point (123)[0茨] and considering the 4-fold symmetry of the coordinate axes of the second crystal leads to the following equivalent points in the Euler space:

\begin{tabular}{|c|c|c|}
\hline$(h k l)$ & {$[u v w]$} & Indices \\
\hline (123) & [032] & $\leq 3$ \\
\hline $\begin{array}{l}\{123\} \\
\{032\} \\
\{032\}\end{array}$ & $\begin{array}{c}<1323> \\
<123> \\
<1323>\end{array}$ & $\begin{array}{l}>3 \\
\leq 3 \\
>3\end{array}$ \\
\hline 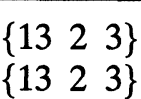 & $\begin{array}{l}<123> \\
<032>\end{array}$ & $\begin{array}{l}>3 \\
>3\end{array}$ \\
\hline
\end{tabular}

Of course not all of these points must necessarily lie within the partial space $P_{1}$. In the maps in Figures $1 \mathrm{a}$ and $\mathrm{b}$ points exhibiting plane indices $>3$ were omitted for the sake of legibility. 


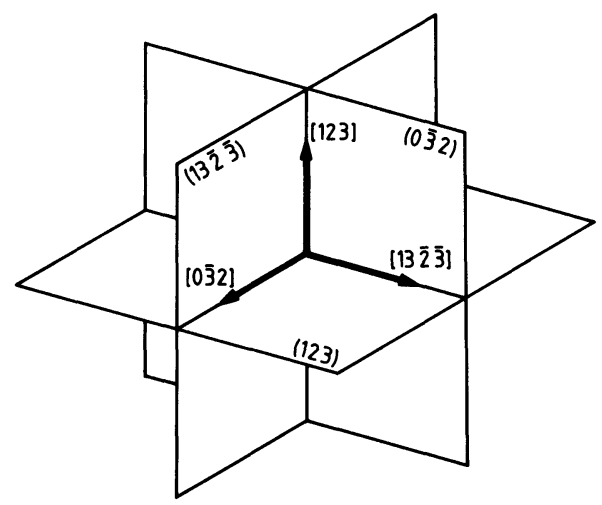

Figure 2 On the selection of equivalent planes and directions in cubic crystals.

\section{APPLICATION OF THE MAPS}

The maps, proposed in this paper, can be used in the following manner: A measured orientation relationship can be plotted as a point in the Euler space. If such a point lies in the vicinity of a point of the maps, the measured orientation relationship can be characterized by the indices $(h k l)[u v w]$ of this special point. If a series of measured orientation relationships is plotted as points into the Euler space, cumulations of points in the vicinity of a point $(h \mathrm{kl})$ $[u v w]$ of the maps indicate that this kind of special orientation relationship occurs preferentially in the considered material.

As an example Figures $3 a-c$ show the orientation relationships measured between neighbouring crystallites in two-phase brasses (rolled and recrystallized) represented as points in the Euler space. Since most of the points are situated between two sections $\varphi_{2}=$ constant, they are plotted in the nearest section. In the case of $\alpha / \alpha$-neighbours (Figure 3a) a moderate accumulation can be seen which can be described by the indices $\{100\}\langle 100\rangle$. Accumulation of points is also evident for $\alpha / \beta$-neighbours (Figure $3 b$ ) according to the special orientation relationships $\{110\}\langle 100\rangle$ and $\{110\}$ $\langle 110\rangle$. 

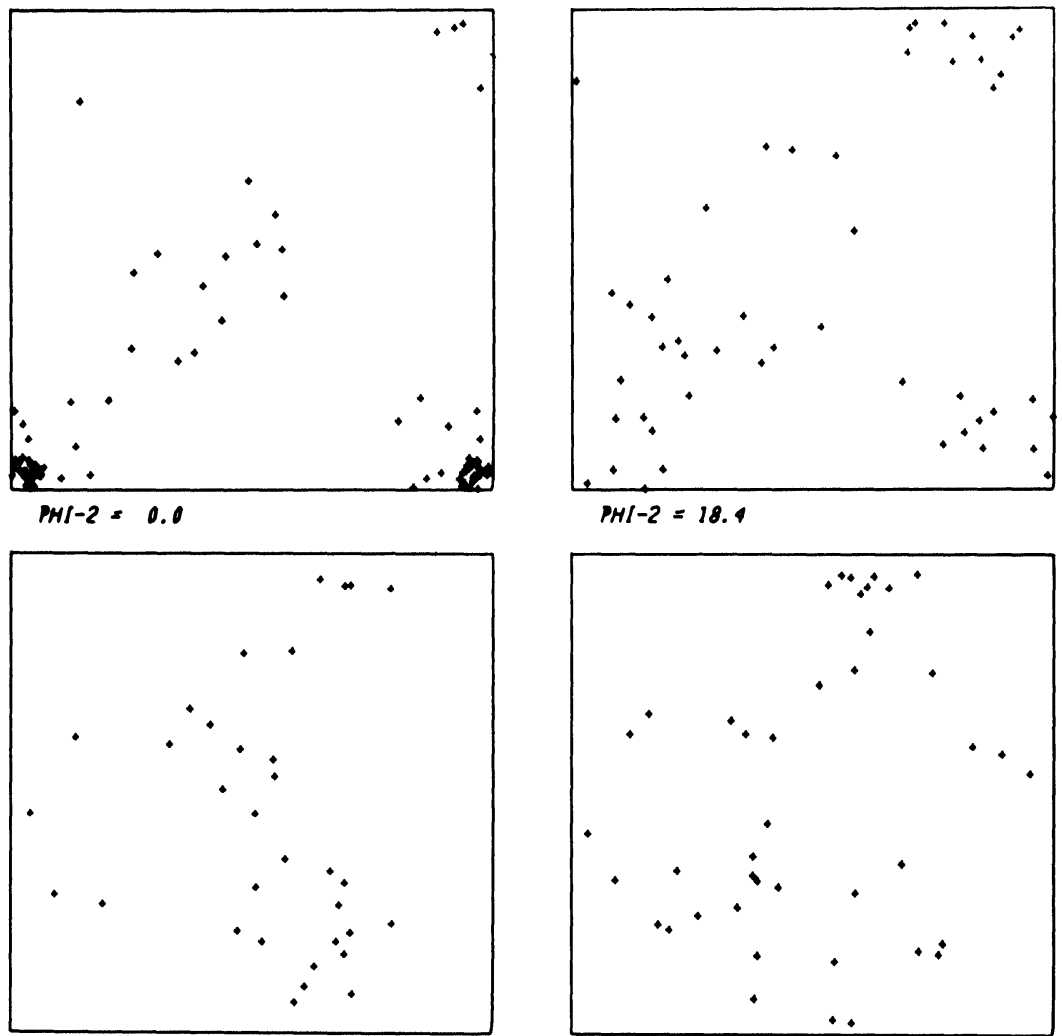

$P H I-2=26.6$

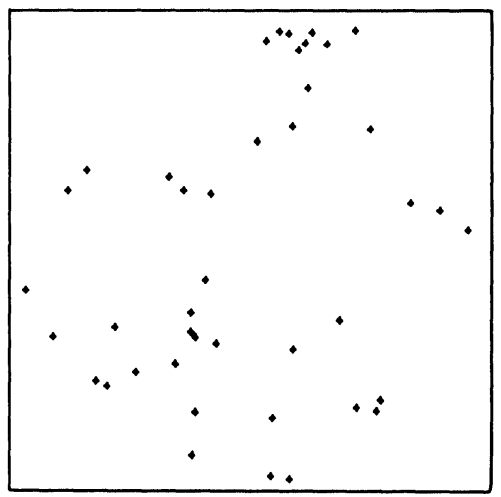

$P H I-2=33.7$
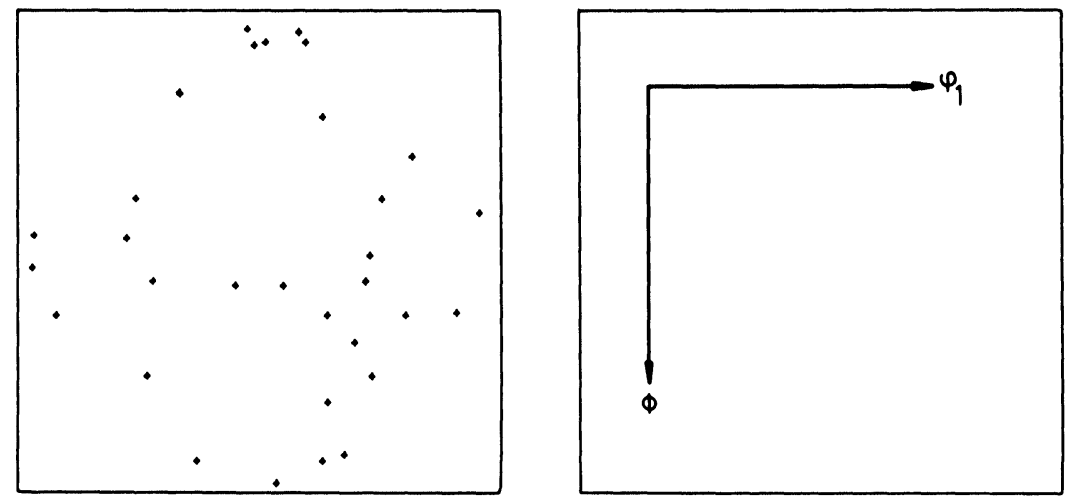

$P H I-2=45.0$

Figure 3a Distribution of measured orientation relationships between $\alpha / \alpha$ neighbours in brass. 


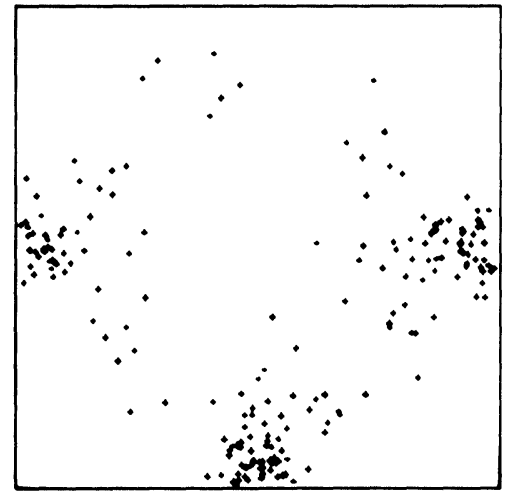

$P H I-2=0.0$

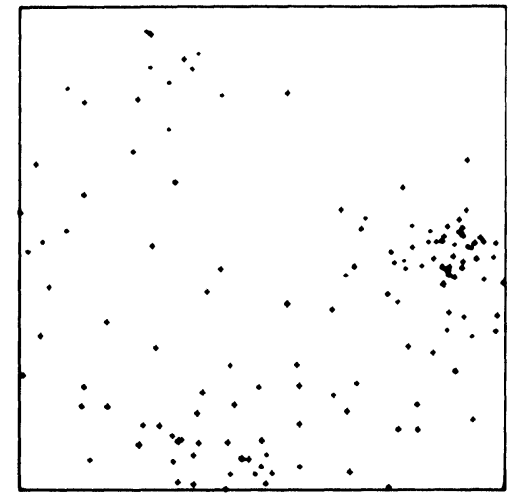

PHI-2 = 18.4

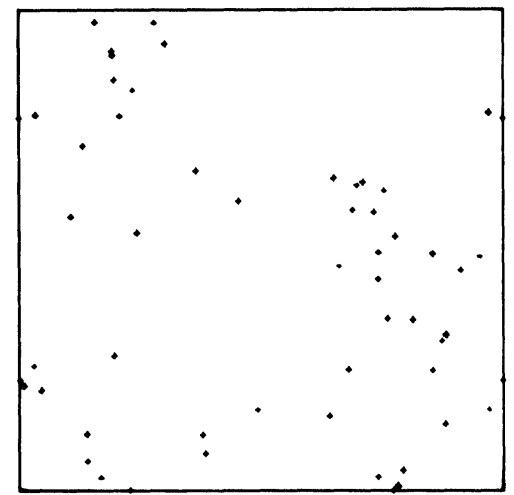

$P N I-2=26.6$

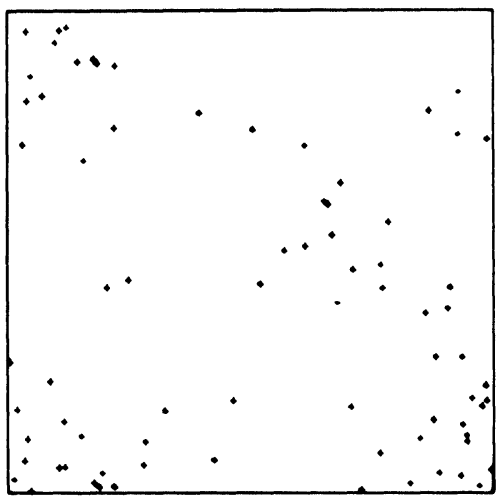

$P H I-2=33.7$
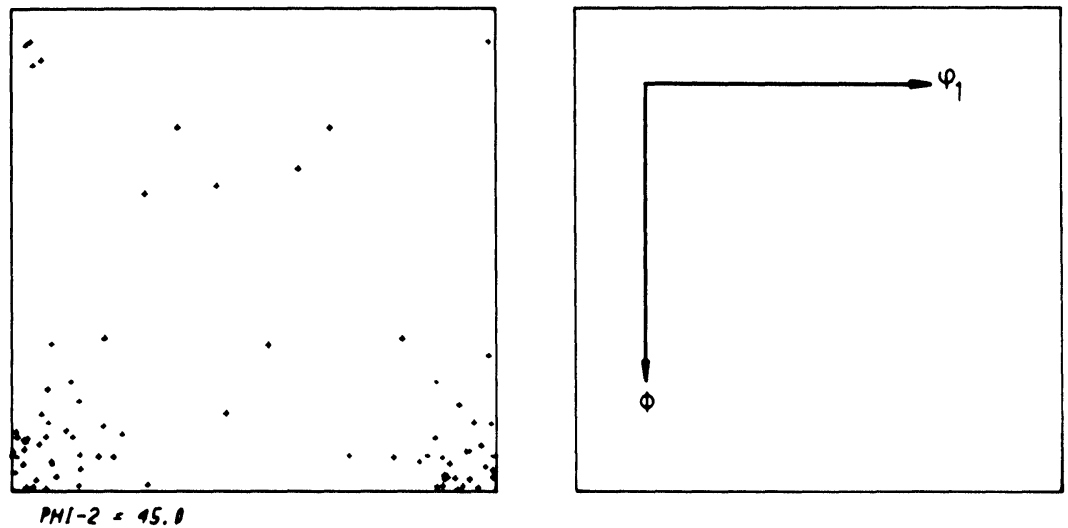

Figure 3b Distribution of measured orientation relationships between $\alpha / \beta$ neighbours in brass. 


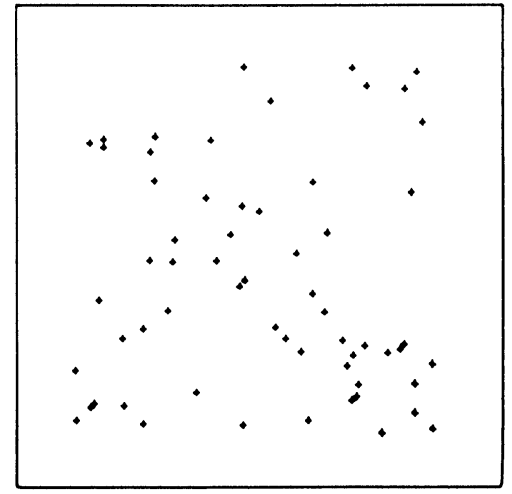

$P H I-2=0.0$

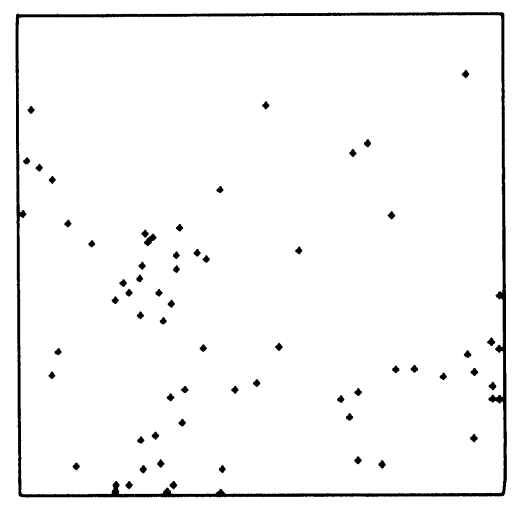

$P H I-2=26.6$

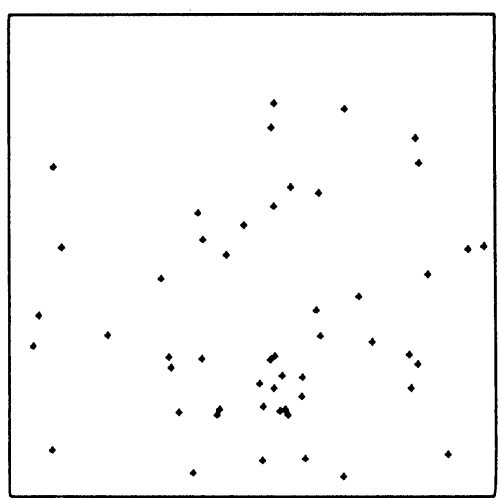

$P H I-2=45.0$

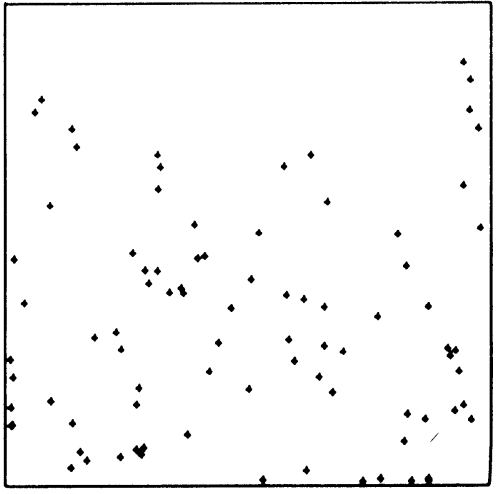

$B H I-2=18.4$

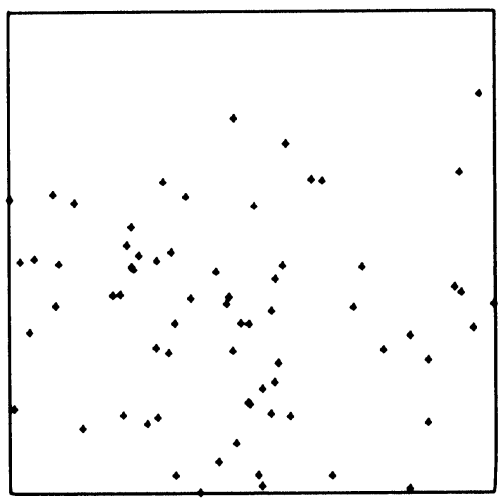

$P H I-2=33.7$

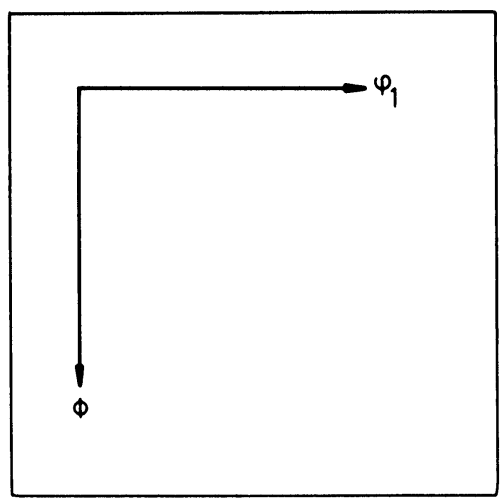

Figure 3c Distribution of measured orientation relationships between $\beta / \beta$ neighbours in brass. 


\section{References}

Bunge, H. J. (1982) Texture Analysis in Material Science. Mathematical Methods, Butterworths, London.

Schläfer, D. and Bunge, H. J. (1974). VII Konferencja Naukowo-Techniczna: Rentfenowska Analiza Strukturalna w Metalurgii i Metaloznawstwie, Gliwice. Werner, E. and Prantl, W. (1988). J. Appl. Cryst., in print. 